\title{
VIABILIDADE DA CULTURA DO MORANGUEIRO NO PARANÁ - BR ${ }^{1}$
}

\author{
EDSON ROBERTO VAZ RONQUE², MAURÍCIO URSI VENTURA ${ }^{3}$, DIMAS SOARES JÚNIOR ${ }^{4}$, \\ ROGÉRIO BARBOSA MACEDO ${ }^{5}$, BRUCE ROBERTO SCHEIDL CAMPOS ${ }^{6}$
}

RESUMO - Avaliou-se a viabilidade da exploração do morangueiro, no Paraná, em duas áreas, 0,3 ha (área média cultivada pela agricultura familiar) e 1 ha, por um ano. Elaborou-se uma planilha de fluxo de caixa a partir da qual se calcularam: Período de Recuperação do Capital (PRC), Retorno sobre Investimento (RI), Valor Presente Líquido (VPL) e Taxa Interna de Retorno (TIR). Todos para Custo Operacional Efetivo (COE), Custo Operacional Total (COT) e Custo Total de Produção (CTP). Para uma situação considerada normal, os indicadores de rentabilidade calculados foram (Tipo de custo: VPL área-padrão (TIR área-padrão) / VPL área efetiva (TIR área efetiva)) COE: US\$ 17.856,55 (42\%) / US\$ 4.795,85 (39\%); COT: US\$ 5.182,40 $(11 \%)$ / - US\$ 1.691,97 (-13\%); CPT: US\$ 4.846,26 (10\%) / -US\$ 1.792,80 (-14\%). Fez-se a análise de cenários para os fatores produtividade, preço de venda e mão de obra, analisando o VPL e a TIR. Verificouse que o tamanho da área influenciou na viabilidade econômica, mostrando a importância de se determinar anualmente a área mínima viável para a agricultura familiar. Os resultados indicaram que a cultura é viável em curto prazo, quando considerado o COE como parâmetro de análise, mas pode não se sustentar em prazos maiores quando se consideram o COT, o CTP e a variação de alguns fatores de produção. Pela análise de cenários definidos pelos fatores de produção analisados, o VPL e a TIR alteram-se para níveis que oferecem risco à exploração.

Termos para indexação: análise econômica da cultura de morango, análise de sensibilidade, produção de morango.

\section{VIABILITY OF THE HOLDING OF THE STRAWBERRY CROP IN PARANA - BR}

\begin{abstract}
We evaluated the viability of the strawberry small farm in Paraná, in two areas, 0.3 ha (average area cultivated by family farms) and 1 ha, during a year. A spreadsheed cash flow was prepared in which it was calculated: Payback Period of Capital (PPC), Return on Investment (ROI), Net Present Value (NPV) and Internal Rate of Return (IRR). All for Operational Cost Effective (OCE), Total Operating Cost (TOC) and Total Cost of Production (TCP). In a situation considered normal, profitability indicators calculated were (Cost Type: NPV standard area (IRR standard area) / NPV effective area (effective area IRR)) OCE: US\$17.856,55 (42\%) / US\$ 4.795,85 (39\%); TOC: US\$ 5.182,40 (11\%) / - US\$ 1.691,97 (-13\%), TCP: US\$ 4.846,26 (10\%) /- US\$1.792,80 (-14\%). A scenario analysis was made for the factors productivity, sales price and manpower, analyzing the NPV and IRR. The size of the area influenced the economic viability, showing the importance of determining annually the minimum viable area for family farms. The results indicated that culture is viable in the short term, when considered as a parameter of the OCE analysis, but can not be sustained in longer when considering the TOC, TCP and variation of some factors of production. By analysis of scenarios defined by production factors, NPV and IRR change to risky levels exploitation.
\end{abstract}

Index terms: economic analysis of the strawberry's culture, sensitivity analysis, production of strawberry.

\footnotetext{
${ }^{1}$ (Trabalho 004-13). Recebido em: 03-01-2013. Aceito para publicação em: 02-10-2013.

${ }^{2}$ Engenheiro agrônomo, Mestrando do Programa de Pós-Graduação, Universidade Estadual de Londrina - UEL. Instituto Paranaense de Assistência Técnica e Extensão Rural - EMATER. E-mail: edsonronque@emater.pr.gov.br.

${ }^{3}$ Engenheiro agrônomo, Mestre e Doutor. Professor, Universidade Estadual de Londrina - UEL. E-mail: mventura@uel.br.

${ }^{4}$ Engenheiro agrônomo, Mestre. Doutorando do Programa de Pós-Graduação, Universidade Estadual de Londrina - UEL. Pesquisador, Instituto Agronômico do Paraná - IAPAR. E-mail: dimasjr@iapar.br.

${ }^{5}$ Engenheiro agrônomo, Mestre e Doutor. Professor, Universidade Estadual do norte do Paraná - UENP. E-mail: macedo@ffalm.br.

${ }^{6}$ Acadêmico de Economia, Universidade Estadual de Londrina - UEL. E-mail: brucescheidl@gmail.com.
} 


\section{INTRODUÇÃO}

A cultura do morangueiro no Estado do Paraná ocupou, na safra 2010, uma área de 535 hectares com produção de 14,38 mil toneladas, representando aproximadamente $7 \%$ do valor bruto da produção - VBP, da fruticultura do Estado (SEAB, 2013). É explorada principalmente por pequenos proprietários e arrendatários e, em alguns municípios, tem grande importância na geração de renda. Sua demanda de mão de obra é elevada, em torno de 15 pessoas/ha/ ano, destacando-se como fixadora de pessoas no campo. A comercialização é feita principalmente nas Centrais de Abastecimento de Curitiba e Londrina, diretamente pelo produtor ou com intermediários locais. Em menor escala, a comercialização também é feita em indústrias de polpas e em supermercados.

Estimativas de custos de produção têm assumido importância crescente na análise da eficiência em processos específicos da produção, indicando o sucesso de determinada empresa em seu esforço de produzir (MARTIN et al., 1994). Ainda que nas propriedades exploradas em regime familiar no $\mathrm{Pa}-$ raná sejam pouco utilizadas, podem ser importante instrumento administrativo, possibilitando identificar mais facilmente fatores de risco, viabilidade de investimentos, possibilitando a elaboração de planejamentos e análises de projetos mais realistas. Considerando as características da cadeia produtiva do morango, o conhecimento dos riscos e dos custos envolvidos em seu processo produtivo pode possibilitar a diminuição dos mesmos.

Agricultores familiares necessitam de explorações que ofereçam satisfatório rendimento em pequenas áreas e também diversificar sua propriedade, para maior segurança econômica e racionalização no uso da mão de obra. A extensão rural oficial do Paraná, executada pelo Instituto EMATER, propõe-se atualmente para a agricultura familiar o desenvolvimento com sustentabilidade, sendo que a cultura do morangueiro é uma das alternativas. Como demonstrado por Schonwald et al. (2008), em determinadas situações, como em estudo sobre a viabilidade de sistemas de irrigação para a agricultura familiar, em diversas explorações, a cultura do morangueiro mostrou-se vantajosa em comparação a outras opções.

Porém, técnicos prestadores de assistência técnica, da extensão rural oficial e privados, e também produtores, consideram a cultura do morangueiro de alto custo, com grande suscetibilidade a problemas fitossanitários e a fatores climáticos adversos, com potencial de danos ao meio ambiente e a oscilações de mercado, e, sendo assim, o conhecimento da viabilidade econômica torna-se um fator de grande importância na decisão do agricultor em investir nesta exploração. Permite, também, ajustes para a manutenção dos benefícios sociais e econômicos a esta categoria, geralmente vulnerável economicamente. Como estudos econômicos sobre o morangueiro no País são poucos, e os conduzidos em outros países estão em condições muito diferentes de produção e mercado, como se pode perceber em Brzozowski e Zmarlicki (2010), produtores e técnicos dispõem de condições precárias para dimensionar seus riscos e sua viabilidade, além de ser comum a crença entre os produtores de que a cultura do morangueiro é viável, sendo importante o aferimento desta crença para maior estabilidade da propriedade familiar, adequando-a ao risco inerente à exploração.

Assim sendo, o objetivo deste estudo é avaliar a viabilidade econômica da exploração do morangueiro pela agricultura familiar no Estado do Paraná, por meio da "Análise de cenários", usando como critérios de avaliação a definição do "Período de recuperação do capital-PRC", do "Retorno sobre o Investimento - RI", da "Taxa Interna de Retorno TIR" e do "Valor Presente Líquido - VPL".

\section{MATERIAIS E MÉTODOS}

Os resultados apresentados são para uma área, denominada padrão, de um hectare, para facilitar correlações e comparações; e também para outra de 0,3 hectare denominada efetiva, por ser a área de cultivo estimada da maioria dos agricultores familiares do Estado do Paraná, baseada pelos dados da SEAB referentes à área e à produção do Estado.

O estudo foi realizado no Estado do Paraná, Brasil, durante a safra de 2010, utilizando-se de dados do mercado das principais regiões produtoras: nordeste do Paraná, Metropolitana de Curitiba e entorno de Imbituva. Os principais municípios produtores na região nordeste são Jaboti, Pinhalão, Japira, Conselheiro Mairinck e Santana do Itararé; na região metropolitana de Curitiba, são Araucária, São José dos Pinhais e Lapa; e na terceira região, são Imbituva e Prudentópolis.

Os dados para a elaboração do orçamento, do fluxo de caixa e da estimativa de demanda da mão de obra foram disponibilizados pelo Instituto Paranaense de Assistência Técnica e Extensão Rural (EMATER), sendo obtidos e utilizados pelos profissionais que prestam assistência técnica e atualizados para a safra de 2010 , sendo eles: 
a) Programa Regional de Tratamento Fitossanitário para Morangueiro para o Nordeste do Paraná - safra de 2010: utilizado para estimar os custos dos produtos usados no controle de pragas e doenças.

b) Tratamento Nutricional para a Cultura do Morangueiro para a Região Nordeste do Paraná - safra de 2010: empregado para estimativa das quantidades e dos custos dos adubos e corretivos. Os preços dos insumos foram coletados nos fornecedores locais.

Para a elaboração do orçamento (estimativa de custo) e do fluxo de caixa, empregou-se a metodologia desenvolvida pelo Instituto de Economia Agrícola do Estado de São Paulo - IEA (MATSUNAGA et al.,1976), com as devidas adaptações para a cultura do morangueiro, calculando-se o Custo Operacional Efetivo (COE), o Custo Operacional Total (COT) e o Custo Total de Produção (CTP). $\mathrm{O}$ uso desta metodologia permite ao produtor decidir se é vantajoso ou não continuar na exploração em curto prazo.

Os custos da mão de obra considerados são os praticados nas regiões produtoras e têm como base o valor e a variação do salário mínimo, e, para os cálculos de mecanização, foram considerados os preços pagos pelos produtores, apurados junto aos prestadores de serviços. A estimativa dos preços recebidos considerou a série histórica de preços médios nominais mensais recebidos pelos produtores do Paraná, disponibilizada pelo Departamento de Economia Rural (DERAL) - SEAB, safras de 2006 a 2010, deflacionados para janeiro de 2010 pelo Índice Geral de Preços - disponibilidade interna (IGP-DI) da Fundação Getúlio Vargas. Utilizaram-se as médias dos preços de setembro, quando se verifica a maior produção.

Considerou-se a produção total dividida em $85 \%$ de morangos embalados para consumo in natu$r a, 13 \%$ destinados à indústria e $2 \%$ de perdas, sendo esta a proporção observada nas lavouras sob condições climáticas, fitossanitárias e de manejo normais.

Considerou-se a dimensão tempo como um ano/safra, visto que é o período predominante de exploração da cultura no Estado do Paraná, uma vez que a condução por mais de um ciclo ainda é incipiente e com potencial de risco agronômico maior. Na região nordeste do Estado, um dos polos de produção considerados na análise, até o momento, todos os produtores renovam a cultura a cada safra. Devido à grande oscilação do mercado e sua dinâmica, foi organizado para a construção do fluxo de caixa, o ciclo de entradas (receitas) e saídas (despesas) em bimestres (Figura 1), sendo que esta adaptação é adequada à cultura do morangueiro, visto que seu ciclo produtivo pode ser dividido em "floradas" de aproximadamente dois meses cada, e que o produtor analisa seu investimento na cultura anualmente, decidindo se irá explorar a cultura a cada ano. A porcentagem da produção total considerada para cada florada foi de $15 \%$ para a primeira, $45 \%$ para a segunda, $25 \%$ para a terceira e $15 \%$ para a quarta, sendo esta a proporção observada nas lavouras sob condições climáticas, fitossanitárias e de manejo normais.

Os indicadores utilizados foram o Valor Presente Líquido (VPL) e a Taxa Interna de Retorno (TIR), por serem métodos que usam fatores de desconto e consideram a dimensão tempo. Analisaram-se a TIR e o VPL, para os três custos, CTP, COT e COE. Para análise de viabilidade, além dos citados anteriormente, foram utilizados também Período de Recuperação do Capital (PRC) e Retorno sobre Investimento (RI). O indicador PRC, embora apresente limitações, é ainda utilizado por muitos agricultores pela sua simplicidade. $\mathrm{O}$ indicador $\mathbf{R I}$, apesar de apresentar limitações, como não considerar a dimensão tempo, é relevante para os objetivos deste trabalho, visto que o tempo considerado é curto o suficiente para permitir tal análise. Para estes dois indicadores, não foi realizada a análise de cenários. Para os métodos VPL e TIR, adotou-se o início do ano como data focal de comparação dos fluxos de caixa e utilizou-se, para o cálculo da TIR, uma taxa suposta de $10 \%$ (padrão do software utilizado para os cálculos).

$\mathrm{Na}$ Análise de Cenários, consideraram-se as variáveis "produtividade", "preço do morango pago ao produtor" e "preço da mão de obra", isto porque as duas primeiras apresentam grande variação durante $o$ período de exploração, sendo que a variação da produtividade se deve ao período de colheita ser longo e a do preço pago ao produtor, devido à quantidade ofertada de morango, não ser uniforme durante o ano, causando grande variação de preços. Quanto ao preço da mão de obra foi devido à disponibilidade desta ser baixa, e esse ser um fator limitante para a exploração da cultura. Cada uma teve sua variação analisada de forma independente da outra. A amplitude das variáveis para a análise de cenários foi a seguinte:

Para a produtividade: foram considerados três níveis, sendo um denominado de normal, a de $44 \mathrm{t} / \mathrm{ha}$ (800 g/planta), e para análise de cenários, uma variação de $25 \%$ para mais e para menos, sendo que, para simulação de ocorrência de problemas agroclimáticos e/ou fitossanitários, que diminuiriam a produtividade, considerou-se a produtividade de $33 \mathrm{t} / \mathrm{ha}$ (600 g/planta), denominada de inferior, e para a simulação de condições agroclimáticas e/ou 
fitossanitárias muito favoráveis, que aumentariam a produtividade, considerou-se a produtividade de $55 \mathrm{t} / \mathrm{ha}$ (1.000 g/planta), denominada de superior.

Para o preço pago ao produtor: pela caixa com $1,2 \mathrm{~kg}^{1}$ de morangos, foram utilizadas as médias dos preços do mês de setembro, quando se verifica a maior produção (pico de safra), levantados pelo DERAL - SEAB, nas safras de 2006 a 2010. Consideraram-se três níveis: normal, o preço médio dos meses de setembro de 2006 a 2010, de US\$ 1,972 ( $\mathrm{R} \$ 3,39)$; inferior, o preço de US\$ 1,58 ( $\mathrm{R} \$ 2,72)$, observado em 2010, simulando uma redução de $\mathbf{1 9 , 7 6 \%}$ frente à média; e superior, o preço de US\$ 2,37 (R\$ 4,09) observado em 2006, simulando um aumento de $\mathbf{2 0 , 6 4 \%}$ frente à média, todos corrigidos pelo IGP-DI da FGV. Já para o preço médio do morango destinado a indústria, foi considerado o preço de US\$ 0,87/ kg (R\$ 1,50), coletado na indústria local, para o qual não foram consideradas variações na análise de cenários realizadas.

Para o custo da mão de obra: foram considerados apenas dois níveis, o denominado normal, que consta no orçamento da safra de 2010 , e outro estimado e denominado superior, com um aumento real de $\mathbf{6 , 5 \%}$, baseado na expectativa de aumento do PIB em 2010 e na política de correção do Salário Mínimo, que estabelece, além da reposição das perdas inflacionárias, um ganho real baseado no crescimento do PIB no período anterior. Assim, em 2011, o Salário Mínimo deve ter um ganho real próximo ao indicado, com provável aumento nas diárias rurais.

A taxa mínima de atratividade (TMA) considerada foi a taxa de juros da caderneta de poupança, de $6 \%$ ao ano, por ser a aplicação mais utilizada pelos produtores. Na Tabela 1, pode-se ver o impacto dos componentes do custo; nas tabelas 2 e 3 , podem-se ver os itens do custo organizados por categorias e a composição do COE, do COT e do CTP. Na Tabela 4 , pode-se ver o fluxo de caixa utilizado, corrigido para valor presente, pela TMA.

\section{RESULTADOS E DISCUSSÃO}

Os indicadores Período de Recuperação do Capital (PRC) (Tabela 5) e Retorno sobre Investimento (RI) (Tabela 6) mostraram que o tamanho da área explorada influencia nos resultados, pois quando se consideram o COT e o CTP para a área efetiva (0,3 ha), não se recupera o capital investido, mesmo no cenário quando se consideram os fatores de produção como normais. Sugere-se que haja uma área de exploração mínima, viável para todos os custos considerados, e, dependendo destes custos, tal área pode variar. Sugere-se ainda que o preço de venda considerado (US\$1,97 (R \$ 3,39)) ou menor, não tenha sido suficiente para evitar saldos negativos. Tal fato é importante para os produtores familiares que cultivam pequenas áreas, visto que normalmente analisam somente o COE, não percebendo esta situação, mesmo com preços médios ou baixos. No entanto, é importante considerar que, no restante da safra, os preços normalmente são mais altos, embora a quantidade de morango produzida seja menor. Provavelmente, a utilização de um preço médio anual, obtido por média ponderada, mostraria um preço maior que o considerado.

Indicadores que utilizam fatores de descontos e consideram a dimensão tempo permitem identificar situações favoráveis e desfavoráveis de investimentos, facilitando a construção e a implementação de estratégias de intervenção (ATALLAH et al., 2012), quando se faz juntamente a análise de cenários dos fatores de produção passíveis de variação, sendo assim utilizado para mensurar resultados econômicos de diversas explorações, quando submetidas a manejos variáveis (KUMAR et. al., 2012).

Os indicadores Valor Presente Líquido (VPL) (Tabela 7) e Taxa Interna de Retorno (TIR) (Tabela 8), calculados para situação normal e para situação de variação dos principais fatores de produção (análise de cenários), mostraram que o tamanho da área explorada e a variação dos fatores de produção considerados determinam a viabilidade, visto os resultados negativos do COT e do CTP e o resultado positivo do COE, para a área efetiva ( $0,3 \mathrm{ha})$, e os resultados positivos para os três custos na área-padrão (1 ha). Assim como os indicadores PRC e RI, o VPL e a TIR também sugerem que haja uma área mínima que seja viável para todos os custos considerados, e também que o preço de venda considerado (US\$ 1,97) $(\mathrm{R} \$ 3,39)$ ou menor, não foi suficiente para evitar saldos negativos para a atividade. Dos fatores considerados, o preço de venda foi o mais incisivo e deve influenciar na viabilidade, quando relacionada ao tamanho da lavoura, de forma mais importante. Tal fato é fundamental para as propriedades familiares que cultivam áreas pequenas, visto que normalmente os produtores analisam somente o COE, não percebendo tal situação.

Como ocorre em outros países, produtos oriundos de sistemas de produção certificados, como a produção orgânica (BRZOZOWSKI;

\footnotetext{
${ }^{1}$ Apesar de o peso da caixa informado pelo levantamento do DERAL ser 1,5 kg, os cálculos foram efetuados com o peso comercializado pelos produtores, que normalmente varia de 1,0 a 1,4 kg. Assim optou-se pelo valor médio de 1,2 kg, para maior fidelidade do estudo.

${ }^{2}$ Taxa cambial do dólar americano de 04 de janeiro de 2010: U\$ $1,00=\mathrm{R} \$ 1,721$.
} 
ZMARLICKI, 2010) e a produção integrada, são mais valorizados pelo mercado que os produzidos em sistema convencional, por apresentarem maior qualidade (REGANOLD et al., 2010), podendo ser uma opção para produtores que não apresentem condições para explorar áreas maiores que a área mínima viável, determinada para a produção em sistema convencional.

A exemplo de outras culturas com consumo mais restrito (EVANS; HUNTLEY, 2011), principalmente quando cultivadas por pequenos produtores, a análise de cenários constitui-se de um importante instrumento para a análise do risco e da viabilidade. A análise de cenários indicou que a ordem dos fatores com maior influência nos resultados foram:

a) situação de baixa: o preço de venda apresenta maior impacto, com maior potencial de prejuízos, sendo que, considerando o $\mathrm{COE}$, única situação com resultado positivo, proporciona um lucro menor, e considerando o COT e o CTP, situações com resultados negativos, proporcionam um prejuízo maior, sendo seguido pela produtividade. A condição de lucros para COE e de prejuízos para COT e CTP foi igual para ambas as áreas.

b) situação de alta: o preço de venda e o preço da mão de obra apresentam maior impacto, com maior potencial de lucros quando aumenta o preço de venda, nos três custos considerados em ambas as áreas, e com situação de prejuízo quando aumenta o preço da mão de obra, considerando o COT e o CTP, na área efetiva (0,3 ha). Mesmo com o aumento do custo da mão de obra, considerando-se o $\mathrm{COE}$, o resultado foi de lucro para ambas as áreas, embora menor do que observado para os outros fatores. Para o fator produtividade, considerando-se o COT e o CTP, o resultado mostrou uma situação-limite de viabilidade. A condição de lucro, considerando-se o COT e o CTP, manteve-se para a área-padrão, mas, para a área efetiva, apenas o aumento do preço de venda proporcionou lucro.

Com relação aos custos, considerando-se o COE, a cultura é viável em curto prazo, para todas as áreas e variações e, quando se consideram o COT e o CTP, a cultura pode não sustentar-se por prazos maiores. Considerando-se o COT, nas situações de baixa, a cultura é inviável para ambas as áreas; nas situações normais, é inviável para área efetiva e viável para área-padrão; e nas situações de alta, é inviável para área efetiva com mão de obra em alta e viável para ambas as áreas nos outros fatores. Quanto ao CTP, nas situações em baixa, a cultura é inviável para ambas as áreas; na situação normal, é inviável para área efetiva e viável para área-padrão; e nas situações em alta, é viável para área-padrão e área efetiva com preços em alta, e inviável para área efetiva com produtividade e mão de obra em alta.

Mesmo a TIR e o VPL demonstrando nas situações com resultados positivos que a exploração é rentável, mostrou também pelos resultados negativos que a exploração pode apresentar prejuízos consideráveis, mesmo com a variação de apenas uma variável, o que, junto com a grande variação do VPL e da TIR, demonstra certo risco de exploração para a agricultura familiar, categoria normalmente frágil economicamente. A TIR referente à área efetiva $(0,3$ ha), com COT, simulada com produção alta (55 t/ha), é igual a TMA, portanto a situação de é viabilidade mínima.

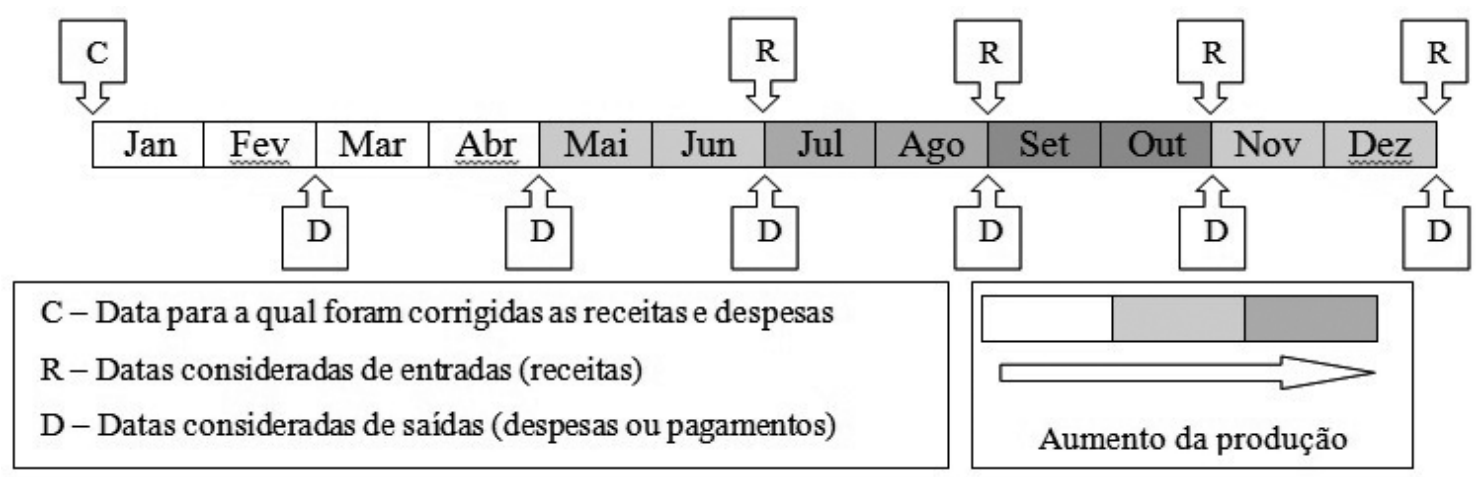

FIGURA 1- Indicação das receitas, despesas e data de correção do fluxo de caixa. 
TABELA 1- Impacto dos itens formadores do custo.

\begin{tabular}{|c|c|}
\hline Descrição dos serviços & $\%$ do CTP \\
\hline Distribuição de calcário + pulverização de herbicidas & 0,26 \\
\hline Aração + gradagem niveladora & 0,42 \\
\hline Canteiramento & 1,53 \\
\hline Fretes - da produção e cobertura morta & 1,48 \\
\hline Subtotal: serviços & 3,69 \\
\hline Descrição da ocupação da mão de obra & \% do CTP \\
\hline Distribuição e incorporação de adubos (orgânico e químicos) & 0,54 \\
\hline Colocação de mulching & 1,15 \\
\hline Limpeza e tratamento de mudas/marcação/plantio & 2,04 \\
\hline Corte de cobertura morta e cobertura de carreadores & 0,83 \\
\hline Capinas, limpeza e toalete da lavoura & 3,04 \\
\hline Pulverizações de agrotóxicos e produtos biológicos & 2,10 \\
\hline Manejo geral da irrigação e fertirrigação & 1,58 \\
\hline Colheita e embalagem & 19,25 \\
\hline Subtotal: mão de obra & $\mathbf{3 0 , 5 3}$ \\
\hline Descrição dos insumos & \% do CTP \\
\hline Mudas + frete & 2,48 \\
\hline Bobinas plásticas $\mathrm{p} /$ mulching & 1,94 \\
\hline Embalagens (cumbucas, caixas e filme plástico) & 23,00 \\
\hline Corretivo, fertilizantes (orgânico e químicos) + frete; adubos foliares & 9,48 \\
\hline Defensivos químicos e biológicos & 6,72 \\
\hline Subtotal: insumos & 43,62 \\
\hline Descrição e depreciação dos equipamentos & \% do CTP \\
\hline Túnel baixo & 6,28 \\
\hline Irrigação por gotejamento & 2,12 \\
\hline Sistema de pulverização - elétrico & 0,26 \\
\hline Subtotal: depreciação dos equipamentos & 8,66 \\
\hline Descrição dos outros custos & \% do CTP \\
\hline CESSR - Contribuição Especial para a Seguridade Social Rural & 2,40 \\
\hline Assistência técnica & 2,18 \\
\hline Taxa FUNRURAL & 2,51 \\
\hline Gerenciamento do empreendimento: & 5,84 \\
\hline Subtotal: outros custos & 12,93 \\
\hline Custo de oportunidade do uso da terra & \% do CTP \\
\hline Custo de oportunidade do uso da terra & 0,57 \\
\hline Subtotal: custo de oportunidade de uso da terra & $\mathbf{0 , 5 7}$ \\
\hline TOTAL & 100 \\
\hline
\end{tabular}



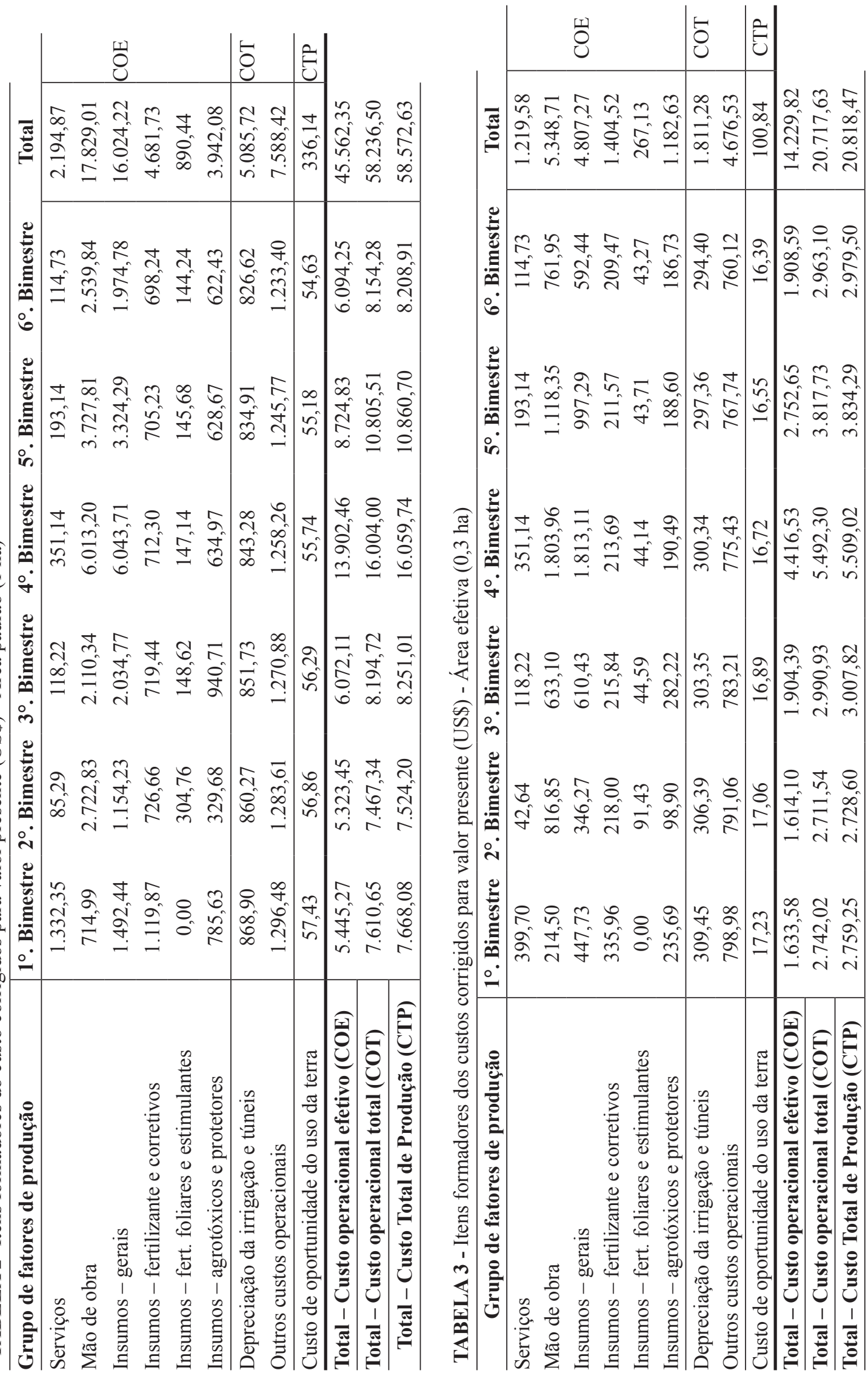
TABELA 4- Fluxo de caixa corrigido para valor presente (US\$).

\begin{tabular}{c|c|c|c|c|c|c|cc}
\hline \multirow{2}{*}{ Período } & \multicolumn{2}{|c|}{ Receita } & \multicolumn{2}{c|}{ CTP1 $^{1}$} & \multicolumn{2}{c|}{ COT $^{2}$} & \multicolumn{2}{c}{ COE $^{3}$} \\
\cline { 2 - 9 } & \multicolumn{2}{|c|}{ Área } & \multicolumn{2}{c|}{ Área } & \multicolumn{2}{c}{ Área } & \multicolumn{2}{c}{ Área } \\
\cline { 2 - 9 } & padrão & efetiva & padrão & efetiva & padrão & efetiva & padrão & efetiva \\
\hline $1^{\circ}$. Bim. & - & - & $7.668,08$ & $2.759,25$ & $7.610,65$ & $2.742,02$ & $5.445,27$ & $1.633,58$ \\
$2^{\circ}$. Bim. & - & - & $7.524,20$ & $2.728,60$ & $7.467,34$ & $2.711,54$ & $5.323,45$ & $1.614,10$ \\
$3^{\circ}$. Bim. & $9.646,21$ & $2.893,86$ & $8.251,01$ & $3.007,82$ & $8.194,72$ & $2.990,93$ & $6.072,11$ & $1.904,39$ \\
$4^{\circ}$. Bim. & $28.651,40$ & $8.595,42$ & $16.059,74$ & $5.509,02$ & $16.004,00$ & $5.492,30$ & $13.902,46$ & $4.416,53$ \\
$5^{\circ}$. Bim. & $15.759,46$ & $4.727,84$ & $10.860,70$ & $3.834,29$ & $10.805,51$ & $3.817,73$ & $8.724,83$ & $2.752,65$ \\
$6^{\circ}$. Bim. & $9.361,82$ & $2.808,55$ & $8.208,92$ & $2.979,50$ & $8.154,28$ & $2.963,10$ & $6.094,25$ & $1.908,59$ \\
\hline
\end{tabular}

$\begin{array}{llllllllll}\text { TOTAL } & 63.418,90 & 19.025,67 & 58.572,63 & 20.818,48 & 58.236,50 & 20.717,63 & 45.562,35 & 14.229,82\end{array}$

${ }^{(1)} \mathbf{C T P}$ - Custo Total de Produção, ${ }^{(2)} \mathbf{C O T}-$ Custo Operacional Total, ${ }^{(3)} \mathbf{C O E}$ - Custo Operacional Efetivo.

TABELA 5- Período de recuperação do capital (PRC).

\begin{tabular}{lcc}
\hline \multicolumn{1}{c}{ Custos } & Área-padrão (1 ha) & Área efetiva (0,3 ha) \\
\hline $\mathrm{COE}^{1}$ & $4^{\circ}$ bimestre & 4 o bimestre \\
$\mathrm{COT}^{2}$ & 5o bimestre & Não recupera, e tem saldo negativo \\
$\mathrm{CTP}^{3}$ & 5o bimestre $^{\text {o }}$ & Não recupera, e tem saldo negativo \\
\hline${ }^{(1)} \mathbf{C O E}-$ Custo Operacional Efetivo, ${ }^{(2)} \mathbf{C O T}-$ Custo Operacional Total, ${ }^{(3)} \mathbf{C T P}-$ Custo Total de Produção.
\end{tabular}

TABELA 6- Retorno sobre o investimento - RI (US\$).

\begin{tabular}{lcccc}
\hline Custos & \multicolumn{2}{c}{ Área padrão (1 ha) } & \multicolumn{2}{c}{ Área efetiva (0,3 ha) } \\
\hline & $\mathrm{RI}$ & $\mathrm{R}^{4}$ & $\mathrm{RI}$ & $\mathrm{R}^{4}$ \\
\hline $\mathrm{COE}^{1}$ & $40,10 \%$ & $18.964,94$ & $34,54 \%$ & $5.103,63$ \\
$\mathrm{COT}^{2}$ & $9,67 \%$ & $5.842,39$ & $-7,51 \%$ & $-1.613,71$ \\
$\mathrm{CTP}^{3}$ & $9,04 \%$ & $5.494,36$ & $-7,96 \%$ & $-1.718,12$ \\
\hline
\end{tabular}

${ }^{(1)} \mathbf{C O E}$ - Custo Operacional Efetivo, ${ }^{(2)} \mathbf{C O T}$ - Custo Operacional Total, ${ }^{(3)} \mathbf{C T P}$ - Custo Total de Produção.

${ }^{(4)} \mathrm{R}$ : Resultado.

TABELA 7- Valor Presente Líquido - VPL (US\$).

\begin{tabular}{l|c|c|c|c|c|c}
\hline & \multicolumn{4}{c}{ Amplitude dos parâmetros considerados } \\
\cline { 2 - 7 } & \multicolumn{2}{c}{ Inferior } & \multicolumn{2}{c}{ Normal } & \multicolumn{2}{c}{ Superior } \\
\hline \multicolumn{1}{c}{ COE $^{1}$} & \multicolumn{2}{|c|}{ Área } & \multicolumn{2}{c}{ Área } & \multicolumn{2}{c}{ Área } \\
\hline \multicolumn{1}{c|}{ Fator } & Padrão & Efetiva & Padrão & Efetiva & Padrão & Efetiva \\
\hline Produtividade & $8.139,59$ & $1.880,76$ & & & $27.573,50$ & $7.710,93$ \\
Preço de venda & $5.322,44$ & $1.035,61$ & $17.856,55$ & $4.795,85$ & $30,951,91$ & $8.724,46$ \\
Custo da mão de obra & - & - & & & $16.697,66$ & $4.448,18$ \\
\hline
\end{tabular}

\begin{tabular}{l|c|c|c|c|c|c}
\hline \multicolumn{1}{c|}{ COT $^{\mathbf{2}}$} & \multicolumn{2}{c|}{ Inferior } & \multicolumn{2}{c|}{ Normal } & \multicolumn{2}{c}{ Superior } \\
\hline \multicolumn{1}{c}{ Fator } & Padrão & Efetiva & Padrão & Efetiva & Padrão & Efetiva \\
\hline Produtividade & $-3.494,59$ & $-4.295,06$ & & & $13.859,40$ & 911,13 \\
Preço de venda & $-7.351,71$ & $-5.452,20$ & $5.182,40$ & $-1.691,97$ & $18.277,77$ & $2.236,65$ \\
Custo da mão de obra & - & - & & & $4.023,51$ & $-2.039,63$ \\
\hline
\end{tabular}

\begin{tabular}{l|c|c|c|c|c|c}
\hline \multicolumn{1}{c|}{ CTP $^{3}$} & \multicolumn{2}{c|}{ Inferior } & \multicolumn{2}{c|}{ Normal } & \multicolumn{2}{c}{ Superior } \\
\hline \multicolumn{1}{c}{ Fator } & Padrão & Efetiva & Padrão & Efetiva & Padrão & Efetiva \\
\hline Produtividade & $-3.830,72$ & $-4.395,90$ & & & $13.523,26$ & 810,30 \\
Preço de venda & $-7.687,84$ & $-5.553,04$ & $4.846,26$ & $-1.792,80$ & $17.941,63$ & $2.135,81$ \\
Custo da mão de obra & - & - & & & $3.687,38$ & $-2.140,47$ \\
\hline
\end{tabular}

${ }^{(1)} \mathbf{C O E}$ - Custo Operacional Efetivo, ${ }^{(2)} \mathbf{C O T}$ - Custo Operacional Total, ${ }^{(3)} \mathbf{C T P}$ - Custo Total de Produção. 
TABELA 8- Taxa Interna de Retorno (TIR).

\begin{tabular}{|c|c|c|c|c|c|c|}
\hline & \multicolumn{6}{|c|}{ Amplitude dos parâmetros considerados } \\
\hline & \multicolumn{2}{|c|}{ Inferior } & \multicolumn{2}{|c|}{ Normal } & \multicolumn{2}{|c|}{ Superior } \\
\hline $\mathrm{COE}^{1}$ & \multicolumn{2}{|c|}{ Área } & \multicolumn{2}{|c|}{ Área } & \multicolumn{2}{|c|}{ Área } \\
\hline Fator & Padrão & Efetiva & Padrão & Efetiva & Padrão & Efetiva \\
\hline Produtividade & $22 \%$ & $18 \%$ & & & $58 \%$ & $55 \%$ \\
\hline Preço de venda & $15 \%$ & $10 \%$ & $42 \%$ & $39 \%$ & $63 \%$ & $61 \%$ \\
\hline Custo da mão de obra & - & - & & & $40 \%$ & $36 \%$ \\
\hline $\mathrm{COT}^{2}$ & \multicolumn{2}{|c|}{ Inferior } & \multicolumn{2}{|c|}{ Normal } & \multicolumn{2}{|c|}{ Superior } \\
\hline Fator & Padrão & Efetiva & Padrão & Efetiva & Padrão & Efetiva \\
\hline Produtividade & $-9 \%$ & $\mathrm{SR}^{4}$ & & & $26 \%$ & $6 \%$ \\
\hline Preço de venda & $-23 \%$ & $\mathrm{SR}^{4}$ & $11 \%$ & $-13 \%$ & $33 \%$ & $13 \%$ \\
\hline Custo da mão de obra & - & - & & & $9 \%$ & $-16 \%$ \\
\hline CTP $^{3}$ & \multicolumn{2}{|c|}{ Inferior } & \multicolumn{2}{|c|}{ Normal } & \multicolumn{2}{|c|}{ Superior } \\
\hline Fator & Padrão & Efetiva & Padrão & Efetiva & Padrão & Efetiva \\
\hline Produtividade & $-10 \%$ & $\mathrm{SR}^{4}$ & & & $25 \%$ & $5 \%$ \\
\hline Preço de venda & $-24 \%$ & $\mathrm{SR}^{4}$ & $10 \%$ & $-14 \%$ & $32 \%$ & $13 \%$ \\
\hline Custo da mão de obra & - & - & & & $8 \%$ & $-17 \%$ \\
\hline
\end{tabular}

${ }^{(1)} \mathbf{C O E}$ - Custo Operacional Efetivo, ${ }^{(2)} \mathbf{C O T}$ - Custo Operacional Total, ${ }^{(3)} \mathbf{C T P}$ - Custo Total de Produção.

${ }^{(4)} \mathrm{SR}$ - não se obteve resposta da função TIR, mas o VPL demonstra que seria um resultado negativo.

\section{CONCLUSÕES}

1-O tamanho da área influencia na viabilidade da exploração do morangueiro e sugere a necessidade de se calcular a área mínima viável para a agricultura familiar a cada safra.

2- Considerando-se o Custo Operacional Efetivo, a cultura do morangueiro é viável para as áreas analisadas.

3-Considerando-se o Custo Operacional Total e o Custo Total de Produção, a cultura do morangueiro pode ser inviável, dependendo principalmente da variação dos fatores preço de venda e custo da mão de obra.

4- A exploração da cultura do morangueiro é de risco para a agricultura familiar.

\section{REFERÊNCIAS}

ATALLAH, S. S.; GÓMEZ, M. I.; FUCHS, M.F.; MARTINSON, T. E. Economic Impact of Grapevine Leafroll Disease on Vitis vinifera cv. Cabernet franc in Finger Lakes Vineyards of New York. American Journal of Enology and Viticulture, Davis, v. 63, n. 1, p. 73-79, 2012.
BRZOZOWSKI, P.; ZMARLICKI, K . Economics of Organic Apple and Strawberry Production in Poland in the years 2007-2009. Journal of Fruit and Ornamental Plant Research, Skierniewice,v.18, n.2, p. 255-264, 2010.

EVANS, E. A.; HUNTLEY, J. Economics of Establishing and Producing Pitaya in Southern Florida: A Stochastic Budget Analysis. HortTechnology, Alexandria, v. 21, n. 2, p. 246-251, 2011.

KUMAR, S; SINGH, R.; ASREY, R.; NANGARE, D. D. Techno-economic evaluation of integrating canal water harvesting and drip irrigation for pomegranate production in a dry eco-region. Irrigation and Drainage, Slough, v. 61, n.3, p. 366-374, 2012.

MARTIN, N. B.; SERRA, R.; ANTUNES, J. F. G.; OLIVEIRA, M.D. M.; OKAWA, H.Custos: Sistema de Custo de Produção Agrícola. Informações Econômicas, São Paulo, v. 24, p.97-122, 1994.

MATSUNAGA, M.; BEMELMANS, P. F.; TOLEDO, P. E. N. de; DULLEY, R.D.; OKAWA, H.; PEDROSO, I.A.Metodologia de Custo de Produção utilizada pelo IEA. Agricultura em São Paulo, São Paulo, v.23, p.1, p. 123-139, 1976. 
REGANOLD, J. P.; ANDREWS, P. K.; REEVE, J.R.; CARPENTER-BOGGS, L.; SCHADT, C. W.; ALLDREDGE, J. R.; ROSS, C. F.; DAVIES, N. M.; ZHOU, J. Fruit and soil quality of organic and conventional strawberry agroecosystems. PLoS ONE, Cairo, v.5, n.9, p.12346, 2010.

PARANÁ (Estado). Secretaria de Estado da Agricultura e Abastecimento. Morango - Área e Produção por região administrativa da SEAB - 2007 a 2011. Disponível em: $<$ http://www. agricultura.pr.gov.br/arquivos/File/deral/fru5.pdf> . Acesso em: 06 abr. 2013.
PARANÁ (Estado). Secretaria de Estado da Agricultura e Abastecimento. Produção agropecuária no Paraná. Disponível em: $<\underline{\mathrm{http}: / /}$ www.seab.pr.gov.br/modules/conteudo/conteudo. php?conteudo=137 $>$. Acesso em: 17 abr.2010.

SCHONWALD, C.; SAMPAIO, S.C.; SATO, M.; FRIGO, E. P.; SUSZEK, M.; FRIGO, J. P. Avaliação econômica de sistemas de irrigação em estabelecimentos rurais familiares na região oeste do Paraná. Irriga, Botucatu, v. 13, n. 1, p. 128-138, 2008. 